\title{
Scores Selection for Emotional Speaker Recognition*
}

\author{
Zhenyu Shan and Yingchun Yang*** \\ College of Computer Science and Technology, Zhejiang University, \\ Hangzhou, China, 310027 \\ \{shanzhenyu, yyc\}@zju. edu.cn
}

\begin{abstract}
Emotion variability of the training and testing utterances is one of the largest challenges in speaker recognition. It is a common situation where training data is the neutral speech and testing data is the mixture of neutral and emotional speech. In this paper, we experimentally analyzed the performance of the GMM-based verification system with the utterances in this situation. It reveals that the verification performance improves as the emotion ratio decreases and the scores of neutral features against his/her model are distributed in the upper area than other three scores(neutral against the model of other speakers, and non-neutral speech against the model of himself/herself and other speakers). Based on these, we propose a scores selection method to reduce the emotion ratio of the testing utterance by eliminating the non-neutral features. It is applicable to the GMM-based recognition system without labeling the emotion state in the testing process. The experiments are carried on the MASC Corpus and the performance of the system with scores selection is improved with an EER reduction from $13.52 \%$ to $10.17 \%$.
\end{abstract}

Keywords: Speaker Recognition, Emotional Speech, Feature Selection.

\section{Introduction}

Emotion variability, which means the emotion states mismatch between the training and testing utterances, is one of the dominant factors that affect the performance of speaker recognition. In most papers, such kind of recognition is called emotional speaker recognition or affective speaker recognition. Different emotion states will affect speech production mechanism in different ways, which leads to acoustical changes in his/her speech[3, 7]. These changes will induce the distribution variability of training and testing features, thus aggravate the performance.

In recent years, many researches have been devoted to the emotional speaker recognition. In general, two situations are considered based on the emotion states of the testing/training utterances. In the first situation, all kinds of emotion states in the testing utterances can be found in the training utterances. Scherer[6] presented an

* This work is supported by the foundings: NCET-04-0545, NSFC_60525202/ 60533040, 863 Program 2006AA01Z136, PCSIRT0652, ZPNSF Y106705, National Key Technology R\&D Program (No. 2006BAH02A01).

** Corresponding author. 
effective structured training approach which aims at making the system get familiar with the emotion variation of the user's voice. As to the second, a restriction is added that the training utterances contain only one emotion state(usually neutral), while the testing utterances involve other emotion states. The adding of this restriction makes it unnecessary to ask the registered users to provide emotional speech, which is user friendly in applications. However, it will affect the system performance. To alleviate the negative effects of the restriction, Wu et al.[2] developed feature modification rule to convert neutral features to emotion features which were used to train the emotion model. Shan et al. $[10,11]$ presented the natural-emotion GMM transformation algorithm to obtain the emotion speech distribution from its neutral speech directly. Wu et al.[5] proposed an emotion-dependent score normalization method derived from $\mathrm{H}$ norm to alleviate the effect of speaker-emotion variability. In these methods, the emotion states involved in testing should be available in advance and the testing utterance is of single emotion state.

In most circumstances, the emotional element, if there is any, always lasts ephemeral when users provide testing utterance, since they tend to provide neutral speech for testing in real application(as discussed in [12]). Normally, the emotional testing utterance is a mixture of neutral and emotion speech. A parameter named the emotion ratio is introduced to define the lasting time of emotional element in one sentence. It is calculated as the emotional speech length against the whole length of the sentence. According to this definition, the emotion ratio of testing utterance in the second situation is $100 \%$. And in this new situation, the focus of our research, the emotion ratio is between $0 \%$ and $100 \%$. The utterances of different emotion ratios will affect the recognition performance in different ways. The relationship between the emotion ratio and the system performance is analyzed. Two results are concluded from the experiments: the performance is improved as the emotion ratio decreases and scores of the neutral speech against his/her own model mainly are distributed in the upper area. Based on these, a scores selection method is proposed to reduce the emotion ratio of testing utterances for improving the performance. According to the distribution of the scores, it sets a threshold to eliminate emotional features. Experimental results show that the performance of different emotion ratios is improved by the scores selection method.

The remainder of this paper is organized as follows. In Section 2, the emotional speech corpus and the speaker recognition based on GMM are introduced. In Section 3, the system performance on the testing speech with different emotion ratios is evaluated and four scores distributions are analyzed. In Section 4, the scores selection method is proposed and the experimental results are given. Conclusions are drawn in Section 5.

\section{Corpus and System Description}

\subsection{Emotional Speech Corpus}

An emotional speech database MASC (Mandarin Affective Speech Corpus) is used in our experiments. It is a corpus provided by linguistic data consortium(LDC) and contains recordings of 68 native speakers ( 23 female and 45 male) and five kinds of emotional states: neutral, anger, elation, panic and sadness. Each speaker reads 5 phrases, 20 sentences three times for each emotional state and 2 paragraphs only for neutral. These materials cover all the phonemes in Chinese. It is constructed for prosodic and 
linguistic investigation of emotion expression in Mandarin. Only the sentences in the corpus are applied in the experiments. These sentences include all the phonemes and most common consonant clusters in Mandarin. The types of sentences are: simple statements, a declarative sentence with an enumeration, general questions (yes/no question), alternative questions, imperative sentences, exclamatory sentences, special questions (wh-questions). More details can be found in [4].

\subsection{System Description}

The speaker verification system used for experiments in this paper is based on the traditional GMM-based method [1]. The diagonal 32-order GMM is applied for modeling. The speech is segmented into frames by a 20-ms window progressing at a $10-$ ms frame rate and the 13-dimension MFCCs[8] are extracted from the speech frames. T-norm[9] technique is used to normalize the dissimilarity scores.

\section{Experiments with Different Emotion Ratios}

In this section, two sets of experiments are designed to analyze the influence of different emotion ratios on a GMM-based speaker verification system.

Table 1. The composition of the testing utterances with different emotion ratios

\begin{tabular}{ccccc}
\hline $\begin{array}{l}\text { Emotion } \\
\text { Ratio } \\
(\%)\end{array}$ & $\begin{array}{l}\text { Non-Neutral } \\
\text { Speech Length } \\
\text { (Sentence) }\end{array}$ & $\begin{array}{l}\text { Neutral } \\
\text { Speech Length } \\
\text { (Sentence) }\end{array}$ & $\begin{array}{l}\text { Testing } \\
\text { Length } \\
\text { (Sentence) }\end{array}$ & $\begin{array}{l}\text { Speech } \\
\text { Testing Speech } \\
\text { Number } \\
\text { each speaker }\end{array}$ \\
\hline 0 & 0 & 3 & 3 & 15 \\
20 & 0.6 & 2.4 & 3 & 45 \\
33.3 & 1 & 2 & 3 & 45 \\
50 & 1.5 & 1.5 & 3 & 45 \\
66.6 & 2 & 1 & 3 & 45 \\
80 & 2.4 & 0.6 & 3 & 45 \\
100 & 3 & 0 & 3 & 15 \\
\hline
\end{tabular}

In the first set of experiments, the system performance is evaluated with the testing utterances of different emotion ratios. The training data is the first 5 sentences read for three times and the testing data is the left $\left(15^{*} 3\right)$ sentences, which is applied to compose the testing utterances with different emotion ratios. The emotion ratio of all the non-neutral utterances in the Corpus is viewed as $100 \%$ and the neutral $0 \%$. The testing utterance with certain emotion ratio is obtained by mixing the neutral utterance with anger, elation, panic or sadness one respectively. The details of the testing utterances are shown in Table 1. In the experiment, each sentence in the Corpus is treated as a length unit. The testing utterances contains 3 sentences (units) lasting about 6$12 \mathrm{~s}$. Each utterance is verified against the models of all 68 speakers. The result of the experiments is shown in Fig. 1. The EER rises in direct proportion to the emotion ratio in each emotion state. It indicates that the system achieves a better performance when the emotion ratio is lower. 

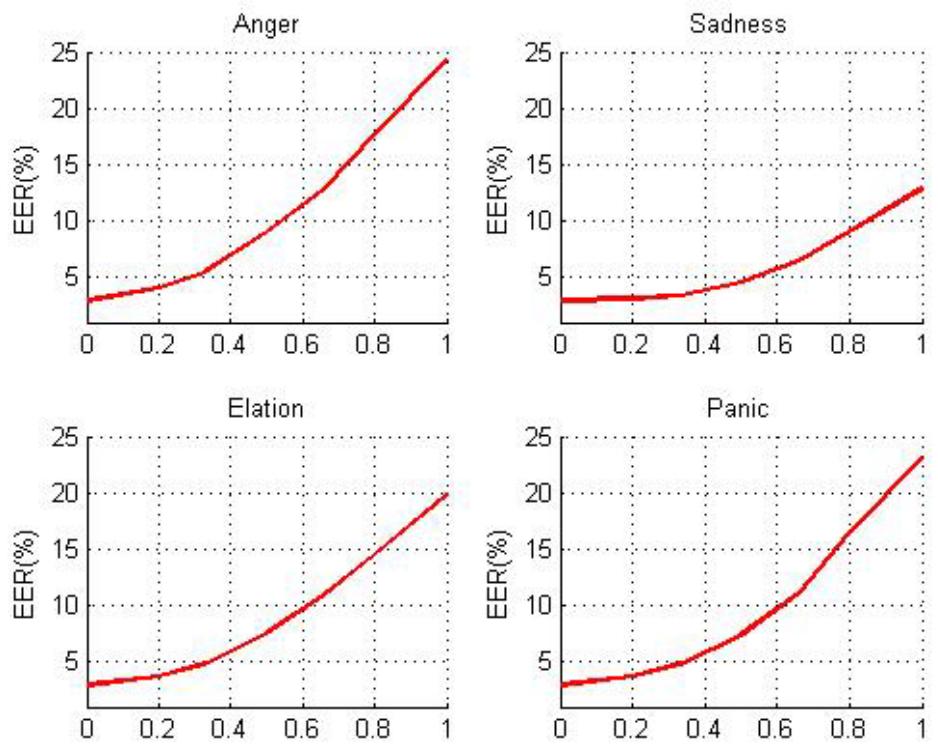

Fig. 1. The performance of emotional speaker recognition with different emotion ratios. $\mathrm{X}$-axis is the emotion ratio and $\mathrm{Y}$-axis is the $\operatorname{EER}(\%)$.

The aim of the second set of experiments is to analyze the relationship between the four types of scores. The first and the second type is the user's neutral and non-neutral speech scored against the model of him/her own respectively $\left(S_{N}, S_{E}\right)$, while the third and the fourth is the neutral and non-neutral speech scored against others' $\operatorname{model}\left(S_{N}\right.$, $\left.S_{E}{ }^{\prime}\right)$. The training utterances are the same as the above experiment and the left utterances of each emotion state are used for scoring. For each speaker, the number of features of each emotion state exceeds 10500 frames. All features are scored against his/her own model and other 67 speakers' models.

The score distribution of one speaker is shown in Fig. 2. It is a typical distribution of the four scores and almost the same for other speakers. To the same speaker, $S_{N}$ is mainly distributed in the upper score area and $S_{E}$ in the relatively lower score area, while the distributions of $S_{N}$ ' and $S_{E}$ ' are similar to each other in the lower area. The mean of all speakers' $S_{N}$ is larger than other three scores and the mean of $\left[\operatorname{avg}\left(S_{N}\right)\right.$ $\left.\operatorname{avg}\left(S_{E}\right)\right]$ is 2.5484. Most speakers (66 of 68) satisfies $\operatorname{avg}\left(S_{E}\right)>\operatorname{avg}\left(S_{N}{ }^{\prime}\right)$ and the mean of $\left[\operatorname{avg}\left(S_{E}\right)-\operatorname{avg}\left(S_{N}{ }^{\prime}\right)\right]$ is 1.4729 . The 47 of all speakers satisfies $\operatorname{avg}\left(S_{N}{ }^{\prime}\right)>\operatorname{avg}\left(S_{E}{ }^{\prime}\right)$ and the left 21 speakers show the opposite. So these results can be concluded as the following formula: $\operatorname{avg}\left(S_{N}\right)>\operatorname{avg}\left(S_{E}\right)>\operatorname{avg}\left(S_{N}{ }^{\prime}\right) \approx \operatorname{avg}\left(S_{E}{ }^{\prime}\right)$. It is a statistical result of the four scores. It can be concluded that the scores of the neutral speech against his/her model are distributed in the highest area. That is to say, most neutral features can be distinguished from his/her emotion features when scoring against his/her model. It also indicates that the system performs better when the utterance is long enough. 


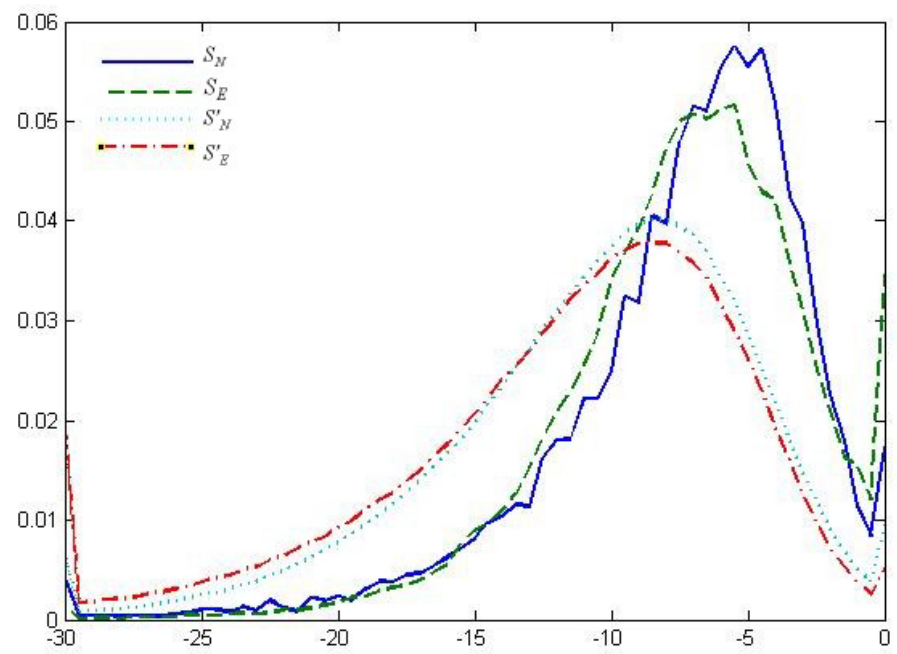

Fig. 2. The 0.5-bin histogram shows the distribution of four types of scores. $\mathrm{X}$-axis is the score value and $\mathrm{Y}$-axis is the possibility.

The results of the experiment elicit two methods to improve the performance of emotional speaker recognition. One is applying the test utterances with low emotion ratio, which suggests the user to provide pure neutral speech for testing or eliminating the non-neutral part from the testing speech. The other is increasing the testing utterances length, yet it can't be satisfied in most applications.

\section{Scores Selection}

\subsection{Method}

In the above section, it can be inferred that decreasing the emotion ratio of the testing utterances is preferable to improve the emotional speaker recognition. Thus, we propose a simple method to distinguish the non-neutral features from the neutral ones in the scoring processing of testing with the purpose to reduce the emotion ratio of testing utterances.

In the GMM-based method, the score is calculated as the average of all testing frames' log-posteriori probability. Formally,

$$
S=\sum_{t=1}^{T} \log G\left(x_{t}\right) / T .
$$

where $G\left(x_{t}\right)$ is the posteriori probability for one feature scored against the GMM and $T$ is the number of feature frames. It only adopts the average of all frames' posteriori probability (scores) for reasoning while their distribution information is ignored. In the above section, we discover that the neutral features can be distinguished from emotion features by their score distribution. 
A scores selection method with two steps is proposed to eliminate the non-neutral features. First, all features' scores and the average of these scores are calculated. Second, the scores higher than the average are selected for the final score computation as in Equation (1). In other words, not all features are effective in the score computation thus some of them are treated as non-neutral features and eliminated to decrease the emotion ratio.

In this method, the eliminated features from the same utterance are different when scored against the different models. When the testing utterance and the model are obtained from the same speaker, more non-neutral features will be eliminated because $\operatorname{avg}\left(S_{N}\right)>\operatorname{avg}\left(S_{E}\right)$. This means that the emotion ratio of testing utterance is reduced. While from different speakers, the eliminated neutral and non-neutral features are almost the same because $\operatorname{avg}\left(S_{N}{ }^{\prime}\right) \approx \operatorname{avg}\left(S_{E}{ }^{\prime}\right)$. Therefore, the emotion ratio changes very little. Though both scores rise up, the score of utterances against his/her own model increases more, so it is advantageous for improving the performance.

\subsection{Performance Evaluation}

One set of experiments was carried out to evaluate the performance of this method. The setting of the experiments was the same as the ones in Section 3. The result of the system with or without scores selection is shown in Fig.3. The system using scores selection outperforms the traditional method in all emotion ratios, which shows this method is effective for speaker recognition with different emotion ratios. Out of our expectation, the performance is also improved when the whole testing utterance only involves neutral or non-neutral state. The performance is markedly improved when the testing utterance is mixed with anger, elation and panic speech, while not obviously better for sadness. The possible reason is that the discrepancy between neutral and sadness features is smaller than other emotion states. It can be found in Fig.1 that the testing utterances blended with sadness speech are the best of the four verification performances. It indicates the score selection method performs better when the nonneutral features are distinguished from neutral ones. In further, all the testing utterances with four emotion states and different emotion ratios are applied for another verification experiment. The result is shown in Fig.4. The performance is improved with the EER reduction from $13.52 \%$ to $10.17 \%$. It indicates its effectiveness for speaker recognition with different emotion ratios.

This experiment is designed to evaluate the effectiveness of the scores selection method by calculating the eliminated non-neutral features. The emotion ratio is reduced after using the selection method as shown in Table 2. When the original emotion ratio is $50 \%$, the emotion ratio deceases to about $43 \%$ after the feature selection. And its outcome is almost the same as the $33 \%$ emotion ratio when not applying it. For sadness, the reduction of emotion ratio is less, so the performance is not so good. This result shows the scores selection method can eliminate the non-neutral features from the testing utterance to decrease the emotion ratio despite it can't remove all non-neutral features.

In the experiment, the average of the testing utterance's scores is used as the threshold. The scores above the average are applied in the final score computation. Based on the rule of scores distribution, the threshold should be set possibly high. However, the reasoning method is on Bayesian theory and its precondition is that there is enough observed data. If the threshold is set too high, there are not enough observed features when the testing utterances length is fixed. Therefore, in the future, further research is still required to get a better threshold. 

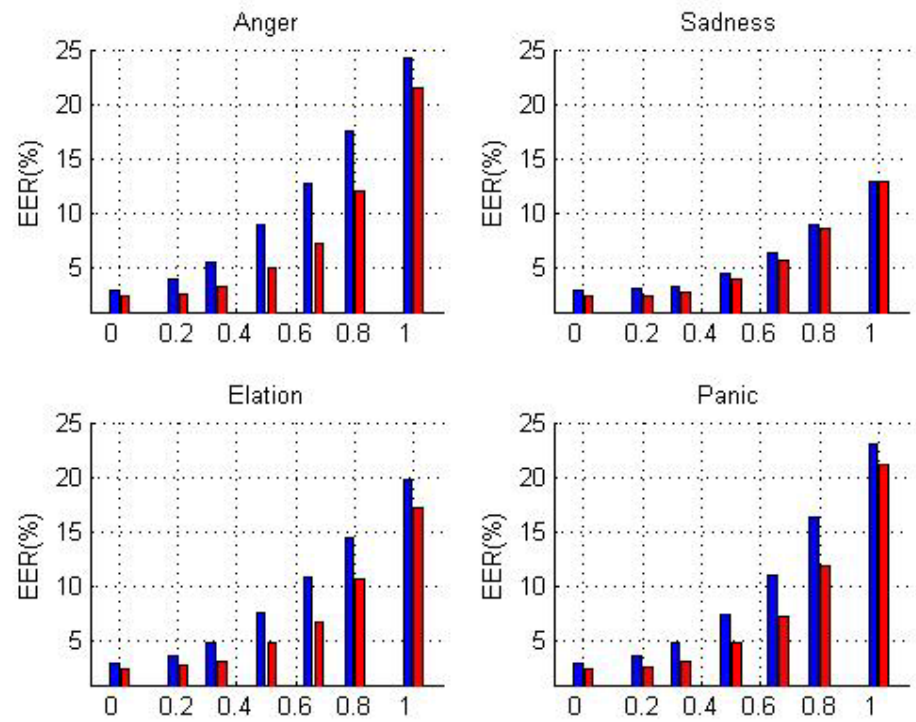

Fig. 3. Comparison of GMM-based system with(right) or without(left) scores selection method in MASC Corpus for four emotion states. $\mathrm{X}$-axis is emotion ratio and $\mathrm{Y}$-axis is EER(\%).

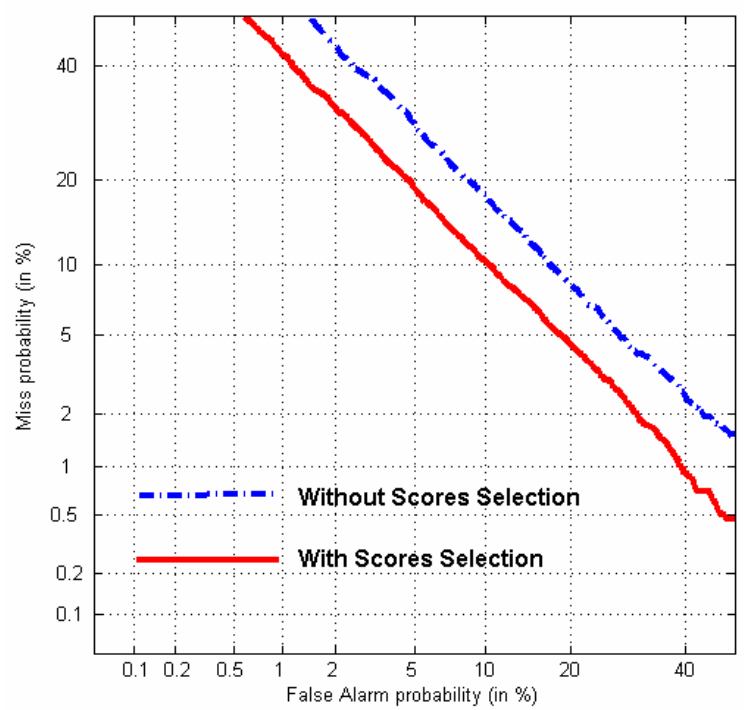

Fig. 4. Comparison of GMM-based system with or without scores selection method in MASC Corpus. The EER of the system with scores selection is $10.17 \%$ and without is $13.52 \%$. 
Table 2. The emotion ratio(ER) after feature selection. The testing utterances and the model are from the same or different speaker. The first/second row is the proportion of the number of changed non-neutral/neutral features against the original features.

\begin{tabular}{lcccc}
\hline & $\begin{array}{c}\text { Anger } \\
\text { same/different }\end{array}$ & $\begin{array}{c}\text { Sadness } \\
\text { same/different }\end{array}$ & $\begin{array}{c}\text { Elation } \\
\text { same/different }\end{array}$ & $\begin{array}{c}\text { Panic } \\
\text { same/different }\end{array}$ \\
\hline Non-Neutral(\%) & $38.41 / 42.69$ & $40.81 / 44.55$ & $40.11 / 43.81$ & $40.71 / 45.43$ \\
Neutral(\%) & $53.27 / 43.66$ & $47.16 / 44.66$ & $51.45 / 47.13$ & $51.32 / 46.48$ \\
ER (original) & $50.00 / 50.00$ & $50.00 / 50.00$ & $50.00 / 50.00$ & $50.00 / 50.00$ \\
ER (changed) & $41.90 / 49.44$ & $46.40 / 49.94$ & $43.81 / 48.17$ & $44.24 / 49.43$ \\
\hline
\end{tabular}

\section{Conclusions}

In the emotional speaker recognition, it is a common situation where users provide neutral utterance mixed with emotion element. In this paper, we analyze the influence of the different emotion ratios on the verification performance and two results are concluded. The first is that the performance of emotional speaker recognition is improved as the emotion ratio declines. The second is that the scores of neutral speech against his/her model are distributed in the upper area, compared with other three scores. The four types of scores are neutral and non-neutral speech against the model of himself/herself and other speakers. On the basis of the results, a scores selection method is proposed to eliminate the non-neutral element from testing speech. The experiment on MASC shows that this method can eliminate the non-neutral features from the testing utterance to decrease the emotion ratio. The system performance is improved with the EER reduction from $13.52 \%$ to $10.17 \%$.

\section{Acknowledgements}

We would like to thank two anonymous referees for their valuable comments and suggestions.

\section{References}

1. Reynolds, D.A., Rose, R.C.: Robust Text-Independent Speaker Identification Using Gasussian Mixture Speaker Models. IEEE Ttransactions on Speech and Audio Processing 3(1), 72-83 (1995)

2. Wu, Z., Li, D., Yang, Y.: Rules Based Feature Modification for Affective Speaker Recognition. In: ICASSP 2006, vol. 1, pp. 661-664 (2006)

3. Scherer, K.R., Johnstone, T., Klasmeyer, G.: Can automatic speaker verification be improved by training the algorithms on emotional speech? In: Proceedings of ICSLP 2000, Beijing, China, pp. 807-810 (2000)

4. Wu, T., Yang, Y., Wu, Z., Li, D.: MASC: A Speech Corpus in Mandarin for Emotion Analysis and Affective Speaker Recognition. In: Odyssey 2006, June 2006, pp. 1-5 (2006)

5. Wu, W., Zheng, T.F., Xu, M.-X., Bao, H.-J.: Study on Speaker Verification on Emotional Speech. In: ICSLP 2006, September 2006, pp. 2102-2105 (2006) 
6. Scherer, K.R.: A cross-cultural investigation of emotion inferences from voice and speech: implication for speech technology. In: Proc. ICSLP 2000 (2000)

7. Scherer, K.R., Johnstone, T., Bänziger, T.: Automatic verification of emotionally stressed speakers: The problem of individual differences. In: Proc. of SPECOM 1998 (1998)

8. Vergin, R., O'Shaughnessy, D., Farhat, A.: Generalized Mel Frequency Cepstral Coefficients for Large-Vocabulary Speaker-Independent Continuous-Speech Recognition. IEEE Ttransactions on Speech and Audio Processing 7(5), 525-532 (1999)

9. Auckenthaler, R., Carey, M., Lloyd-Thomas, H.: Score normalization for text-independent speaker verification systems. Digital Signal Processing 10, 42-54 (2000)

10. Shan, Z., Yang, Y., Wu, Z.: Natural-Emotion GMM Transformation Algorithm for Emotional Speaker Recognition. In: InterSpeech 2007, pp. 782-785 (2007)

11. Shan, Z., Yang, Y.: Polynomial Function Based Neutral-Emotion GMM Transformation for Speaker Recognition. In: ICPR 2008 (2008) (accepted)

12. Shan, Z., Yang, Y., Wu, Z.: SCS: A Speech Check-in System. In: The 8th International Conference on Signal Processing, vol. 4, pp. 752-756 (2006) 